\title{
AN OPTIMAL CONTROL PROBLEM INVOLVING A CLASS OF LINEAR TIME-LAG SYSTEMS
}

\author{
K. L. TEO ${ }^{1}$, K. H. WONG ${ }^{2}$ AND Z. S. WU ${ }^{3}$
}

(Received 10 December 1984; revised 1 April 1985)

\begin{abstract}
A class of convex optimal control problems involving linear hereditary systems with linear control constraints and nonlinear terminal constraints is considered. A result on the existence of an optimal control is proved and a necessary condition for optimality is given. An iterative algorithm is presented for solving the optimal control problem under consideration. The convergence property of the algorithm is also investigated. To test the algorithm, an example is solved.
\end{abstract}

\section{Introduction}

In [10], a feasible directions algorithm has been devised for solving a class of nonlinear time-lag optimal control problems with linear control constraints and nonlinear terminal inequality constraints. This feasible direction algorithm was first initiated by Polak and Mayne in [7] for an optimal control problem involving lumped-parameter system, but without time-delayed arguments. The convergence result of the algorithms reported in [10] and [7] is as follows:

If the sequence of feasible controls generated by the algorithm has an accumulation point in the strong topology of $L_{\infty}$, then it satisfies a necessary condition for optimality.

Clearly, this convergence result is not satisfactory in the sense that there is no guarantee of the existence of such an accumulation point.

\footnotetext{
${ }^{1}$ Department of Industrial and Systems Engineering, National University of Singapore, Kent Ridge, Singapore 0511, Republic of Singapore.

${ }^{2}$ Department of Applied Mathematics, University of Witwatersrand, 1, Jan Smuts Avenue, Johannesberg 2001, South Africa.

${ }^{3}$ Department of Mathematics, Zhongshan University, Guangzhou, China.

๑ Copyright Australian Mathematical Society 1986, Serial-fee code 0334-2700/86
} 
The main aim of this paper is to obtain a more natural convergence result than those of [10] and [7]. More precisely, we shall show that the sequence of feasible controls generated by the feasible directions algorithm has an accumulation point in the weak* topology of $L_{\infty}$. Furthermore, each of these accumulation points is shown to satisfy a necessary condition for optimality. However, this is possible only for a special case of [10]. More precisely, our result is only valid for the case in which the dynamic system is linear.

To demonstrate the efficiency of the algorithm, an example is solved.

\section{The problem statement}

Consider the following delay-differential equation defined on the fixed time interval $(0, T]$ :

$$
\dot{x}(t)=\sum_{j=0}^{s}\left\{A_{j}(t) x\left(t-h_{j}\right)+B_{j}(t) u\left(t-h_{j}\right)\right\},
$$

where

$$
x \equiv\left[x_{1}, \ldots, x_{n}\right]^{T} \in R^{n}, \quad u \equiv\left[u_{1}, \ldots, u_{r}\right]^{T} \in R^{r}
$$

are, respectively, the state and the control vectors; the superscript $T$ denotes the transpose; $A_{j}$ and $B_{j}$ are, respectively, $n \times n$ and $n \times r$ matrix-valued functions defined on $\left[0, T+h_{s}\right]$. The $h_{j}$ are time delays, ordered so that

$$
0=h_{0}<h_{1} \cdots<h_{s}<T ; \quad s<\infty .
$$

The initial function for the differential equation (1a) is

$$
x(t)=\phi(t), \quad t \in\left[-h_{s}, 0\right) ; \quad x(0)=\Gamma,
$$

where $\phi(t) \equiv\left[\phi_{1}, \ldots, \phi_{n}\right]^{T}$ is a given piecewise continuous function from $\left[-h_{s}, 0\right)$ into $R^{n}$, and $\Gamma$ is a given vector in $R^{n}$.

Let $U$ be a compact and convex subset of $R^{r}$ such that for all $u \in U$,

$$
E_{i}^{T} u \leqslant b_{i}, \quad i=1, \ldots, p,
$$

where $E_{i}, i=1, \ldots, p$, are $r$-vectors and $b_{i}, i=1, \ldots, p$, are real numbers. Let $\beta:\left[-h_{s}, 0\right) \rightarrow R^{r}$ be a given piecewise continuous function. Let $\mathscr{U}$ be the class of all admissible controls defined by

$\mathscr{U} \equiv\left\{u: u \equiv\left[u_{1}, \ldots, u_{r}\right]^{T}\right.$ is a measurable function defined on $\left[-h_{s}, T\right]$ such that $u(t) \in U$ for all $t \in[0, T]$ and $u(t)=\beta(t)$ for all $t \in$ $\left.\left[-h_{s}, 0\right)\right\}$. 
Let $\langle\cdot, \cdot\rangle$ and $|\cdot|$ denote, respectively, the usual inner product and norm in a Euclidean space. For any

$$
n \times r \text { matrix } A \equiv\left\{A_{i, J}\right\}_{\substack{i=1, \ldots, n \\ j-1, \ldots, r}}
$$

let

$$
\|A\|=\sum_{j=1}^{r} \sum_{i=1}^{n}\left|A_{i, j}\right| \text {. }
$$

Let $L_{\infty}^{q}$ (resp. $L q$ ) denote the Banach space $L_{\infty}\left(\left[-h_{s}, T\right], R^{q}\right)$ (resp. $\left.L_{1}\left(\left[-h_{s}, T\right], R^{q}\right)\right)$ of all essentially bounded measurable functions (resp. of all integrable functions) from $\left[-h_{s}, T\right]$ into $R^{q}$. The norms of $L_{\infty}^{q}$ and $L_{1}^{q}$ are, respectively, defined by

$$
\|u\|_{\infty} \equiv \underset{i \in\left[-h_{s}, T\right]}{\operatorname{ess} \sup }|u(t)|
$$

and

$$
\|u\|_{1} \equiv \int_{-h_{s}}^{T}|u(t)| d t .
$$

For each $u \in L_{\infty}^{r}$, Let $x(u)$ be the corresponding vector-valued function which is absolutely continuous on $(0, T]$ and satisfies the differential equation (1a) almost everywhere on $(0, T]$ and the initial condition (1b) everywhere on $\left[-h_{s}, 0\right]$. This function $x(u)$ is called the solution of the system (1) corresponding to $u \in L_{\infty}^{r}$. In view of Appendix (B1) of [4], pages 104-106, we observe that $x(u)$ can be expressed in terms of the Oguztöreli kernel matrix of the second kind, namely:

$$
\begin{aligned}
x(u)(t)= & N(0, t) \Gamma+\sum_{j=0}^{s} \int_{-h_{j}}^{0}\left\{N\left(\tau+h_{j}, t\right) A_{j}\left(\tau+h_{j}\right) \phi(\tau)\right\} d \tau \\
& +\sum_{j=0}^{s} \int_{0}^{t}\left\{N(\tau, t) B_{j}(\tau) u\left(\tau-h_{j}\right)\right\} d \tau,
\end{aligned}
$$

where $N(\tau, t)$ (the Oguztöreli kernel matrix of the second kind), is the continuous $n \times n$ matrix defined on $[0, T] \times[0, T]$ which satisfies the system

$$
\begin{gathered}
\frac{\partial N(\tau, t)}{\partial t}=\sum_{j=0}^{s} A_{j}(t) N\left(\tau, t-h_{j}\right), \quad t \in[0, T], \quad \tau \in[0, T], \\
N(\tau, \tau)=I \quad \text { (identity matrix), } \\
N(\tau, t)=0 \quad \text { for all } \tau>t .
\end{gathered}
$$

The terminal inequality constraints may now be stated as follows:

$$
J^{m}(u) \equiv g^{m}(x(u)(T)) \leqslant 0, \quad m=1, \ldots, l,
$$

where $g^{m}: R^{n} \rightarrow R, m=1, \ldots, l$, are real-valued functions defined on $R^{n}$. 
Clearly, not all controls from $\mathscr{U}$ will satisfy the constraints (6). Let $\mathscr{F}$ denote the class of all those controls from $\mathscr{U}$ such that the constraints (6) are satisfied. Elements from $\mathscr{F}$ are called feasible controls and $\mathscr{F}$ is called the class of feasible controls. In this paper, we assume that $\mathscr{F}$ is not empty.

Our optimal control problem, denoted by $P$, may now be formally stated as follows:

Subject to the system (1), find a feasible control $u \in \mathscr{F}$ such that the cost functional

$$
J^{0}(u)=g^{0}(x(u)(T))+\int_{0}^{T}\left\{f(t, x(u)(t))+u^{T}(t) M(t) u(t)\right\} d t
$$

is minimized over $\mathscr{F}$.

We assume that the following conditions are satisfied.

(A1) $A_{\jmath}, j=0, \ldots, s$, are real continuous $n \times n$ matrices defined on $\left[0, T+h_{s}\right]$; $B_{j}, j=0, \ldots, s$, are real continuous $n \times r$ matrices defined on $\left[0, T+h_{s}\right]$.

(A2) $M$ is a $r \times r$ symmetric, positive semidefinite continuous matrix defined on $[0, T]$.

(A3) $x \rightarrow g^{m}(x), m=0, \ldots, l,(t, x) \rightarrow f(t, x)$ are continuously differentiable on $R^{n}$ and $[0, T] \times R^{n}$, respectively.

\section{Preparatory results}

For each $u \in L_{\infty}^{r}$, let the functions $\lambda_{m}(u):\left[-h_{s}, T+h_{s}\right] \rightarrow R^{n}, m=0, \ldots, l$, be the solution of the adjoint system

$$
\begin{aligned}
& \lambda_{m}(t)=-\sum_{j=0}^{s} A_{j}^{T}\left(t+h_{j}\right) \lambda_{m}\left(t+h_{j}\right), \quad m=1, \ldots, l, \quad t \in[0, T), \\
& \lambda_{0}(t)=-\sum_{j=0}^{s} A_{j}^{T}\left(t+h_{j}\right) \lambda_{0}\left(t+h_{j}\right)-\nabla_{x} f(t, x(u)(t)), \quad t \in[0, T),
\end{aligned}
$$

with the conditions

$$
\begin{array}{ll}
\lambda_{m}(T)=\nabla_{x} g^{m}(x(u)(T)), & m=0, \ldots, l, \\
\lambda_{m}(t)=0, & m=0, \ldots, l, t \in\left[-h_{s}, 0\right),
\end{array}
$$

and

$$
\lambda_{m}(t)=0, \quad m=0, \ldots, l, t \in\left[T, T+h_{s}\right) .
$$

Following a similar approach as that used in the proof of Theorem 3.1 of [12], it can be shown that the Fréchet derivatives of the functionals $J^{0}, J^{1}, \ldots, J^{l}$ at 
$u^{0} \in L_{\infty}^{r}$ are, respectively,

$$
\begin{aligned}
& J_{u^{0}}^{0}(w)=-\int_{-h_{s}}^{T}\left\{\sum_{j=0}^{s} \lambda_{0}^{T}\left(u^{0}\right)\left(t+h_{\jmath}\right) B_{j}\left(t+h_{j}\right)+2 u^{0^{r}}(t) M(t)\right\} w(t) d t, \\
& J_{u^{0}}^{m}(w)=-\int_{-h_{s}}^{T}\left\{\sum_{j=0}^{s} \lambda_{m}^{T}\left(u^{0}\right)\left(t+h_{j}\right) B_{j}\left(t+h_{j}\right)\right\} w(t) d t, \quad m=1, \ldots, l,
\end{aligned}
$$

for all $w \in L_{\infty}^{r}$.

REMARX 3.1. In view of the problem $P$, we see that the only non-standard feature is the inclusion of the terminal inequality constraints on the state variables. In this situation, one may include the constraints in the Pontryagin theory, by modifying boundary conditions for $\lambda_{0}$ as it is done in [3]. Alternatively, one may treat these constraints as those in mathematical programming problems. The latter approach is adopted in this paper. Thus, we need to calculate the "gradients" of these constraints. To do so, we take each constraint as a "cost functional". Then, by applying the usual Pontryagin theory, we can write down the corresponding "Hamiltonian". On this basis, we obtain readily the corresponding "adjoint system" (cf. 8(a)). The formula for the "gradient" of the constraint concerned is given in (9b). The reader may find more detail of this idea in Appendix V of [11], pages 291-292.

Definition 3.1. Let $\left\{u^{k}\right\}$ be a sequence in $\mathscr{U}$ and let $\bar{u}$ be an element in $\mathscr{U}$. Then, we say that $u^{k} \stackrel{w^{*}}{\rightarrow} \bar{u}$ (or $u^{k}$ converges to $\bar{u}$ in the weak* topology) if

$$
\int_{-h_{s}}^{T} u^{k}(t) v(t) d t \rightarrow \int_{-h_{s}}^{T} \bar{u}(t) v(t) d t
$$

for all $v \in L_{1}^{r}$.

Definition 3.2. Let $\left\{u^{k}\right\}$ be a sequence in $L_{\infty}^{r}$ and let $\bar{u}$ be an element in $L_{\infty}^{r}$. Then we say that $u^{k} \stackrel{w^{*}}{\rightarrow} \bar{u}$ in $L_{\infty}^{r}$ (or $u^{k}$ converges to $\bar{u}$ in the weak ${ }^{*}$ topology in $\left.L_{\infty}^{r}\right)$ if

$$
\int_{-h_{s}}^{T} u^{k}(t) v(t) d t \rightarrow \int_{-h_{s}}^{T} \bar{u}(t) v(t) d t
$$

for all $v \in L_{1}^{r}$.

LEMMA 3.1. Consider the system (1). Let $x$ be considered as a mapping from $\mathscr{U}$ into $L_{\infty}^{n}$, where the norm in the space $L_{\infty}^{n}$ is again denoted by $\|\cdot\|_{\infty}$. Then

(i) There exists a positive constant $C$, independent of $u \in \mathscr{U}$, such that

$$
\|x(u)\|_{\infty} \leqslant C \text {. }
$$


(ii) For each $t \in[0, T]$

$$
\left|x\left(u^{k}\right)(t)-x(\bar{u})(t)\right| \rightarrow 0 \quad \text { whenever } u^{k} \stackrel{w^{*}}{\rightarrow} \bar{u} .
$$

(iii) $\left\|x\left(u^{k}\right)-x(u)\right\|_{\infty} \rightarrow 0$ whenever $u^{k} \stackrel{\omega^{*}}{\rightarrow} \bar{u}$.

Proof. The proof of (i) follows easily from (4), (A1) and the definition of $\mathscr{U}$. The proof of (ii) and (iii) requires (4) and (A1).

LEMMA 3.2. Consider the adjoint system (8). Let $\lambda_{m}$ be considered as mappings from $\mathscr{U}$ into $L_{\infty}^{n}$. Then

(i) For each $m=0, \ldots, l$, there exists a positive constant $C_{m}$, independent of $u \in \mathscr{U}$, such that

$$
\left\|\lambda_{m}(u)\right\|_{\infty} \leqslant C_{m}
$$

(ii) For each $m=0, \ldots, l$,

$$
\left\|\lambda_{m}\left(u^{k}\right)-\lambda_{m}(\bar{u})\right\|_{\infty} \rightarrow 0 \text { whenever } u^{k} \stackrel{w^{*}}{\rightarrow} \bar{u} .
$$

Proof. Using (8b) and (8c), we deduce from Cauchy's inequality that for all $u \in \mathscr{U}$ and for all $t \in[0, T]$,

$$
\begin{aligned}
\left|\lambda_{0}(u)(t)\right| \leqslant & \left|\nabla_{x} g^{0}(x(u)(T))\right|+\int_{t}^{T} \sum_{j=0}^{s}\left\|A_{j}^{T}\left(\tau+h_{j}\right)\right\|\left|\lambda_{0}(u)\left(\tau+h_{j}\right)\right| d \tau \\
& +\int_{t}^{T}\left|\nabla_{x} f(\tau, x(u)(\tau))\right| d \tau
\end{aligned}
$$

where $\|\cdot\|$ is as defined in (3).

Since $x(u)(\cdot)$ is absolutely continuous on $[0, T]$ for each $u \in \mathscr{U}$, it follows from Lemma 3.1 and (A3) that there exists a constant $K_{1}>0$, independent of $u \in \mathscr{U}$, such that

$$
\left|\nabla_{x} g^{0}(x(u)(T))\right| \leqslant K_{1}
$$

and

for all $t \in[0, T]$.

$$
\left|\nabla_{x} f(t, x(u)(t))\right| \leqslant K_{1}
$$

Thus, we deduce from (10), (8e) and (A1) that

$$
\left\|\lambda_{0}(u)\right\|_{\infty} \leqslant K_{2}+K_{3} N_{1}\left(\left\|\lambda_{0}(u)\right\|_{\infty}\right)
$$

where

$$
\begin{aligned}
& K_{2} \equiv K_{1}(T+1), \\
& K_{3} \equiv \max _{\substack{j \in\{0, \ldots, s\} \\
t \in[0, T]}}\left\|A_{j}(t)\right\|
\end{aligned}
$$


and

$$
N_{1}(w)(t)=\int_{t}^{T} w(\tau) d \tau
$$

Now, by using the same argument as that used to obtain the inequality (A4) of [12] (i.e. the inequality (4) in the Appendix of [12]) from the inequality (A2) of [12], we deduce from (11) that

$$
\left\|\lambda_{0}(u)\right\|_{\infty} \leqslant \sum_{i=0}^{\infty} K_{2} T^{i} K_{3}^{\prime} / i ! \equiv C_{0} .
$$

Similarly, for each $m=1, \ldots, l$, there exists a positive constant $C_{m}$ such that

$$
\left\|\lambda_{m}(u)\right\|_{\infty} \leqslant C_{m}
$$

for all $u \in \mathscr{U}$.

It remains to establish the validity of the second part of the lemma. For this, let $\left\{u^{k}\right\}$ be a sequence of controls in $\mathscr{U}$ which converge to $\bar{u}$ in the weak* topology. Then, by using (8b) and (8c), we deduce from Cauchy's inequality that for any integer $k$,

$$
\begin{aligned}
& \left|\lambda_{0}\left(u^{k}\right)(t)-\lambda_{0}(\bar{u})(t)\right| \\
& \leqslant\left|\nabla_{x} g^{0}\left(x\left(u^{k}\right)(T)\right)-\nabla_{x} g^{0}(x(\bar{u})(T))\right| \\
& \quad+\int_{t}^{T} \sum_{j=0}^{s}\left\|A_{j}^{T}\left(\tau+h_{\jmath}\right)\right\|\left|\lambda_{0}\left(u^{k}\right)\left(\tau+h_{\jmath}\right)-\lambda_{0}(\bar{u})\left(\tau+h_{\jmath}\right)\right| d \tau \\
& \quad+\int_{t}^{T}\left|\nabla_{x} f\left(\tau, x\left(u^{k}\right)(\tau)\right)-\nabla_{x} f(\tau, x(\bar{u})(\tau))\right| d \tau
\end{aligned}
$$

From Lemma 3.1 and (A3), we note that for any given $\varepsilon>0$, there exists an integer $\hat{k}>0$ such that for all $k>\hat{k}$,

$$
\left|\nabla_{x} g\left(x\left(u^{k}\right)(T)\right)-\nabla_{x} g(x(\bar{u})(T))\right|<\varepsilon
$$

and

$$
\left|\nabla_{x} f\left(t, x\left(u^{k}\right)(t)\right)-\nabla_{x} f(t, x(\bar{u})(t))\right|<\varepsilon
$$

for all $t \in[0, T]$.

On the basis of (15), it follows from (A1), (16) and (17) that

$$
\left\|\lambda_{0}\left(u^{k}\right)-\lambda_{0}(\bar{u})\right\|_{\infty}<K_{2} \varepsilon+K_{3} N_{1}\left(\left\|\lambda_{0}\left(u^{k}\right)-\lambda_{0}(\bar{u})\right\|_{\infty}\right),
$$

where $K_{2}, K_{3}$ and $N_{1}$ are defined in (12), (13) and (14), respectively.

Then, by using the same argument as that used to obtain the inequality (A4) of [12] from the inequality (A2) of [12], we deduce from (18) that

$$
\left\|\lambda_{0}\left(u^{k}\right)-\lambda_{0}(\bar{u})\right\|_{\infty} \leqslant \sum_{i=0}^{\infty} K_{1} T^{i} K_{2}^{i} \varepsilon / i ! \equiv K_{3} \varepsilon
$$


Therefore

$$
\left\|\lambda_{0}\left(u^{k}\right)-\lambda_{0}(\bar{u})\right\|_{\infty} \rightarrow 0 \text { whenever } u^{k} \stackrel{\omega^{*}}{\rightarrow} \bar{u} .
$$

Similarly, for each $m=1, \ldots, l$, we have

$$
\left\|\lambda_{m}\left(u^{k}\right)-\lambda_{m}(\bar{u})\right\|_{\infty} \rightarrow 0 \text { whenever } u^{k} \stackrel{w^{*}}{\rightarrow} \bar{u} .
$$

This completes the proof of the lemma.

LeMma 3.3. For each $m=0,1, \ldots, l$, and for each $w \in L_{\infty}^{r}, J_{u}^{m}(w)$ is continuous in $u$ with respect to the weak ${ }^{*}$ topology in the sense that

$$
J_{u^{k}}^{m}(w) \rightarrow J_{\bar{u}}^{m}(x) \text { whenever } u^{k} \stackrel{w^{*}}{\rightarrow} \bar{u} .
$$

Proof. The proof follows easily from (9), (A1), (A2) and part (ii) of Lemma 3.2 .

LEMMA 3.4. For each $m=0,1, \ldots, l$, and for each $u \in \mathscr{U}, J_{u}^{m}(w)$ is continuous in $w$ with respect to the weak* topology in $L_{\infty}^{r}$ in the sense that

$$
J_{u}^{m}\left(w^{k}\right) \rightarrow J_{u}^{m}(\bar{w}) \text { whenever } w^{k} \stackrel{w^{*}}{\rightarrow} \bar{w} \text { in } L_{\infty}^{r} .
$$

Proof. The proof follows easily from (9), (A1), (A2) and part (i) of Lemma 3.2.

\section{Existence of optimal control}

In this section, we shall show that the optimal control problem has a solution. More precisely, we have

THEOREM 4.1. If $\mathscr{F}$ is nonempty, then the problem $P$ has a solution.

Proof. From part (i) of Lemma 3.1 and the continuity property of $x(u)(\cdot)$, we can find a compact set $Z$ in $R^{n}$ such that

$$
x(u)(t) \in Z
$$

for all $u \in \mathscr{U}$ and for all $t \in[0, T]$. Now, by using this result, together with the compactness of the set $U$ in $R^{r}$ and the assumptions (A2) and (A3), it can be shown (by method of [9]) that the cost functional $J^{0}(u)$ is bounded on $\mathscr{U}$. Thus,

$$
\inf _{u \in J^{0}} J^{0}(u)=\sigma>-\infty \text {. }
$$


Let $\left\{u^{k}\right\} \subset \mathscr{F}$ be a sequence such that

$$
\lim _{k \rightarrow \infty} J^{0}\left(u^{k}\right)=\sigma .
$$

Since $U$ is a compact and convex subset of $R^{r}, \mathscr{U}$ is sequentially compact in the weak* topology (cf. Theorem I.6.2 of [11], page 31). Thus, there exists a function $u^{*} \in \mathscr{U}$ and a subsequence of the sequence $\left\{u^{k}\right\}$, again indexed by $k$, such that $u^{k} \rightarrow u^{*}$ in the weak* topology as $k \rightarrow \infty$.

It remains to show that $u^{*}$ is an optimal control of the problem $P$. From Lemma 3.1, (A3) and (6), it follows that, for each $m=1, \ldots, l$,

$$
g^{m}\left(x\left(u^{*}\right)(T)\right)=\lim _{k \rightarrow \infty} g^{m}\left(x\left(u^{k}\right)(T)\right) \leqslant 0 .
$$

This means that $u^{*} \in \mathscr{F}$. Again from Lemma 3.1 and (A3), we have

$$
g^{0}\left(x\left(u^{*}\right)(T)\right)=\lim _{k \rightarrow \infty} g^{0}\left(x\left(u^{k}\right)(T)\right)
$$

and

$$
\int_{0}^{T} f\left(t, x\left(u^{*}\right)(t)\right) d t=\lim _{k \rightarrow \infty} \int_{0}^{T} f\left(t, x\left(u^{k}\right)(t)\right) d t .
$$

From (A2), it is clear that the function $u^{T}(t) M(t) u(t)$ is convex with respect to $u$. Thus, from Theorem 1.2.14 of [1], page 26, we have

$$
\liminf _{k \rightarrow \infty} \int_{0}^{T}\left(u^{k}\right)^{T}(t) M(t) u^{k}(t) d t \geqslant \int_{0}^{T} u^{* T}(t) M(t) u^{*}(t) d t .
$$

Now, by virtue of (7), it follows from (19), (20) and (21) that

$$
\sigma=\liminf _{k \rightarrow \infty} J^{0}\left(u^{k}\right) \geqslant J^{0}\left(u^{*}\right) .
$$

Recall that the sequence $\left\{u^{k}\right\} \subset \mathscr{F}$ is chosen such that

$$
\lim _{k \rightarrow \infty} J^{0}\left(u^{k}\right)=\inf _{u \in \mathscr{F}} J^{0}(u)=\sigma .
$$

Thus

$$
\liminf _{k \rightarrow \infty} J^{0}\left(u^{k}\right)=\lim _{k \rightarrow \infty} J^{0}\left(u^{k}\right)=\inf _{u \in \mathscr{F}} J^{0}(u)=\sigma
$$

Hence

$$
\inf _{u \in \mathscr{F}^{*}} J^{0}(u) \geqslant J^{0}\left(u^{*}\right) .
$$

But, we have already shown that

Thus it is clear that

$$
u^{*} \in \mathscr{F}
$$

$$
J^{0}\left(u^{*}\right)=\inf _{u \in \mathscr{F}} J^{0}\left(u^{0}\right)
$$

Thus, in turn, implies that $u^{*} \in \mathscr{F}$ is an optimal control for the problem $P$, as there could not be any other feasible control which will make $J^{0}$ smaller than $\inf _{u \in F} J^{0}(u)$, and hence $J\left(u^{*}\right)$. Thus, the proof is complete. 
REMARK 4.1. By examining the arguments given for Theorem 4.1, it is easy to see that $\mathscr{F}$ is sequentially compact in the weak* topology.

\section{Subproblem}

For the problem $P$, we want to minimize the cost functional $J^{0}(u)$, subject to constraints $J^{m}(u) \leqslant 0, m=1, \ldots, l$, which have been built as part of the adjoint system (i.e. (8a) and (8c)-(8e)). We shall do this by a "feasible direction" method similar to that of [10] (and hence [7]). Thus, at each iteration of the algorithm, it is required to calculate a search direction which decreases $J^{0}$, while pointing into the region specified by (6). Each of these search directions corresponds to a subproblem which is itself an optimal problem and can be solved by a standard convex program (Meyer-Polak proximity algorithm, see [6], pages 234-238). In what follows, we shall formulate the subproblem and describe an algorithm to solve it.

For any $\varepsilon>0$ and $u \in \mathscr{F}$, let $I_{\varepsilon}(u)$ denote the $\varepsilon$-active index set, i.e.

$$
I_{\varepsilon}(u) \equiv\{0\} \cup\left\{m \in\{1, \ldots, l\}: J^{m}(u) \geqslant-\varepsilon\right\} .
$$

for any subset $I$ of the set $\{0,1, \ldots, l\}$, let $\psi_{I}: \mathscr{F} \rightarrow R$ be defined by

$$
\psi_{I}(u)=\min _{w \in \mathscr{U}-u} \max _{m \in I}\left\{J_{u}^{m}(w)\right\}
$$

where

$$
\begin{aligned}
\mathscr{U}-u & \equiv\{v-u: v \in \mathscr{U}\} \\
& \equiv\{v: v \in \mathscr{U}\}-u .
\end{aligned}
$$

In view of Lemma 4.1 of [13], it is clear that for each $u \in \mathscr{F}$, there exists a $w_{I}^{u} \in \mathscr{U}-u$ such that

$$
\psi_{I}(u)=\max _{m \in I}\left\{J_{u}^{m}\left(w_{I}^{u}\right)\right\} .
$$

The subproblem can now be stated as:

Problem (Ps). For each $u \in \mathscr{F}$ and $\varepsilon>0$, find a search direction $w_{\varepsilon}^{u} \in \mathscr{U}-u$ which minimizes

$$
\max _{m \in I_{e}(u)}\left\{J_{u}^{m}(w)\right\}
$$

i.e.,

$$
\begin{aligned}
\psi_{I_{\varepsilon}(u)}(u) & =\min _{w \in \mathscr{U}-u} \max _{m \in I_{\varepsilon}(u)}\left\{J_{u}^{m}(w)\right\} \\
& =\max _{m \in I_{\varepsilon}(u)}\left\{J_{u}^{m}\left(w_{\varepsilon}^{u}\right)\right\} .
\end{aligned}
$$


To present an algorithm for solving this problem, we need some preparation.

Let $n_{\varepsilon}^{u}$ denote the cardinality of the set $I_{\varepsilon}^{u}$ and let $I_{\varepsilon}^{u} \equiv\{i(1), i(2), \ldots, i(q)\}$, where $q \equiv n_{\varepsilon}^{u}$ and $i(1), \ldots, i(q)$ are arranged in the natural order with $i(1)=0$.

Let

$$
C_{\varepsilon}^{u}=\left\{\xi=\left[\xi_{1}, \ldots, \xi_{q}\right]^{T}: \xi_{k}=J_{u}^{\prime(k)}(w), k=1, \ldots, q, w \in \mathscr{U}-u\right\} .
$$

Since $\mathscr{U}-u$ is convex and compact in the weak* topology, it follows from Lemma 3.4 that $C_{e}^{u}$ is a compact and convex subset of $R^{q}$.

The Meyer-Polask proximity algorithm requires a subprocedure for finding a point $\xi_{\varepsilon, c}^{u} \in C_{\varepsilon}^{u}$ which minimizes $\langle c, \xi\rangle$ subject to $\xi \in C_{\varepsilon}^{u}$ for each $c \equiv$ $\left[c_{1}, \ldots, c_{q}\right]^{T} \in R^{q}$. From (9) and (23), this is equivalent to finding a search direction $w_{e, c}^{u} \in \mathscr{U}-u$ which minimizes

$$
\left\{\sum_{j=0}^{s} \sum_{k=1}^{q} c_{k} \lambda_{i(k)}^{r}(u)\left(t+h_{j}\right) B_{j}\left(t+h_{j}\right)+2 c_{1} u^{T}(t) M(t)\right\} w(t)
$$

over all $w \in \mathscr{U}-u$ and for all $t \in\left[-h_{s}, T\right]$, where $\lambda_{t(k)}, k=1, \ldots, q$, is the solution of the corresponding system (8).

LEMMA 5.1. Let $c$ be a given constant vector in $R^{q}$, where $q \equiv n_{\varepsilon}^{u}$. Then, for each $u \in \mathscr{F}$, there exists a $w_{\varepsilon, c}^{u} \in \mathscr{U}-u$ such that

$$
\min _{w \in \mathscr{Q}-u}\left\{\sum_{j=0}^{s} \sum_{k=1}^{q} c_{k} \lambda_{t(k)}^{T}(u)\left(t+h_{j}\right) B_{j}\left(t+h_{j}\right)+2 c_{1} u^{T}(t) M(t)\right\} w(t)
$$

(i)

$$
\begin{aligned}
& =\left\{\sum_{j=0}^{s} \sum_{k=1}^{q} c_{k} \lambda_{t(k)}^{T}(u)\left(t+h_{j}\right) B_{j}\left(t+h_{J}\right)+2 c_{1} u^{T}(t) M(t)\right\} w_{e, c}^{u}(t) \\
& \text { for all } t \in\left[-h_{s}, T\right] . \\
& \left\langle c, \xi_{e, c}^{u}\right\rangle \leqslant\langle c, \xi\rangle
\end{aligned}
$$

(ii) for all $\xi \in C_{e}^{u}$, where $\xi_{e, c}^{u} \equiv\left[\left(\xi_{\varepsilon, c}^{u}\right)_{1}, \ldots,\left(\xi_{\varepsilon, c}^{u}\right)_{q}\right]^{T}$ with

$$
\left(\xi_{e, c}^{u}\right)_{k} \equiv J^{i(k)}\left(w_{e, c}^{u}\right), k=1, \ldots, q .
$$

Proof. The proof follows as a special case of Lemma 4.1 of [10].

Now, let

$$
D_{e, a}^{u} \equiv\left\{\eta \in R^{n_{\varepsilon}^{u}}: \eta_{j} \in[b,(u, \varepsilon), a]\right\},
$$

where $b(u, \varepsilon) \in R^{q}$ denotes the vector

$$
b(u, \varepsilon) \equiv\left[\left(\xi_{\varepsilon, e_{1}}^{u}\right)_{1},\left(\xi_{\varepsilon, e_{2}}^{u}\right)_{2}, \ldots,\left(\xi_{\varepsilon, e_{q}}^{u}\right)_{q}\right]^{T}
$$

where $q=n_{\varepsilon}^{u}$ and for each $j=1, \ldots, n_{\varepsilon}^{u}, e_{j}$ denotes the $j$ th unit vector in $R^{n_{\varepsilon}^{u}}$. 
In what follows, $\xi$ will denote a point in $C_{\varepsilon}^{u}$ and $\eta$ a point in $D_{\varepsilon, a}^{u}$. Then, the Meyer-Polak proximity algorithm can be used to determine the least value of $a$ such that $C_{\varepsilon}^{u} \cap D_{e, a}^{u} \neq \varnothing$ and to determine a point in the intersection. Let $\hat{\xi}_{\varepsilon}^{u}$ be such a point in the intersection. Then

$$
\psi_{I_{e}(u)}(u)=\max \left\{\left(\hat{\xi}_{e}^{u}\right),: j=1, \ldots, n_{e}^{u}\right\} .
$$

We can now present the Meyer-Polak proximity algorithm for determining $\psi_{I_{e}^{u}}(u)$.

ALGORITHM 5.1.

DATA: $u \in \mathscr{F}, \varepsilon>0, I_{\varepsilon}(u)$.

Step 0. Set $\xi^{0}=0$. Set $w^{0}=0$.

Compute $b=b(u, \varepsilon)$.

Set $\eta^{0}=b$.

Set $a^{0}=\max \left\{b_{j}: j=1,2, \ldots, n_{\varepsilon}^{u}\right\}$.

Set $i=0$.

Step 1.

If $\left|\xi^{i}-\eta^{i}\right|=0$, stop.

Else set $\sigma^{i}=\xi^{i}-\eta^{i}$.

Step 2.

Compute $w_{\varepsilon, \sigma^{\prime}}^{u}$ and $\xi_{\varepsilon, \sigma^{\prime}}^{u}$.

Set $\overline{w^{i}}=w_{e, a^{\prime}}^{u}, \bar{\xi}^{\prime}=\xi_{e, \sigma^{\prime}}^{u}$.

Step 3.

If $\left\langle\sigma^{i}, \bar{\xi}^{i}\right\rangle \leqslant\left\langle\sigma^{i}, \eta^{i}\right\rangle$, set $a^{i+1}=a^{i}, \overline{\eta^{i}}=\eta^{i}$.

Else set $a^{i+1}=\min \left\{a: D_{e, a}^{u} \cap H \neq \varnothing, a \geqslant a^{i}\right\}$, where $H=\left\{y \in R^{n_{e}^{u}}:\left\langle y, \sigma^{i}\right\rangle=\left\langle\bar{\xi}_{i}, \sigma^{i}\right\rangle\right\}$.

Compute any $\overline{\eta^{i}} \in D_{u, a_{i+1}}^{e} \cap H$.

Step 4. Compute $\xi^{i+1} \in\left[\xi^{i}, \overline{\xi^{i}}\right], \eta \in\left[\eta^{i}, \overline{\eta^{i}}\right]$ such that $\left|\xi^{\prime+1}-\eta^{\prime+1}\right|=\min \left\{|\xi-\eta|: \xi \in\left[\xi^{i}, \bar{\xi}\right], \eta \in\left[\eta^{i}, \overline{\eta^{\eta}}\right]\right\}$.

Set $w^{i+1}=w^{i}+\left(\overline{w^{i}}-w^{i}\right)\left|\xi^{i}-\xi^{i+1}\right| / \mid \xi^{i}-\overline{\xi^{i}}$.

Set $i=i+1$.

Go to Step 1.

REMARK 5.1. For each $i, \xi^{i}$, which is a point in $C_{\varepsilon}^{u}$, is generated by $w^{i} \in \mathscr{U}-u$, i.e.

$$
\left(\xi^{i}\right)_{k}=J_{u}^{i(k)}\left(w^{i}\right), \quad i(k) \in I_{\varepsilon}(u) .
$$

REMARK 5.2. From Remark 4.5 of [10], there exists an integer $i_{0}>0$ such that

$$
\max _{k}\left\{\left(\xi^{i_{0}}\right)_{k}\right\}-\max _{k}\left\{\left(\eta^{i_{0}}\right)_{k}\right\} \leqslant \varepsilon / 2
$$


Let $\bar{\psi}_{e}(u), \underline{\psi}_{\varepsilon}(u)$ and $\bar{w}_{\varepsilon}^{u}$ denote $\max _{k}\left\{\left(\xi^{i_{0}}\right)_{k}\right\}, \max _{k}\left\{\left(\eta^{i_{0}}\right)_{k}\right\}$ and $w^{i_{0}}$, respectively. Then $\bar{\psi}_{\varepsilon}^{u}(u), \underline{\psi}_{\varepsilon}(u)$ and $\bar{w}_{e}^{u}$, which can be computed in a finite number of iterations, satisfy

$$
\begin{aligned}
& \psi_{I_{\varepsilon}^{u}}(u) \in\left[\psi_{\varepsilon}(u), \bar{\psi}_{\varepsilon}(u)\right], \\
& \bar{\psi}_{\varepsilon}(u)-\underline{\psi}_{\varepsilon}(u) \leqslant \varepsilon / 2,
\end{aligned}
$$

and

$$
\bar{\psi}_{\varepsilon}(u)=\max _{j \in I_{\varepsilon}^{u}}\left\{J_{u}^{j}\left(\bar{w}_{\varepsilon}^{u}\right)\right\}
$$

\section{An algorithm}

In this section, we shall present a feasible direction algorithm for solving the problem $P$ and study its convergence properties.

\section{Algorithm 6.1.}

DATA: $\alpha, \beta \in(0,1)$ and $\varepsilon^{\prime}>0$.

Step 0. Select a $u^{0} \in \mathscr{F}$; set $i=0$.

Step 1. Set $\varepsilon_{0}=\varepsilon^{\prime}$; set $j=0$.

Step 2. Compute, using Algorithm 5.1, a search direction $\bar{w}_{\varepsilon_{j}}^{u^{\prime}}$, together with $\bar{\psi}_{e,}\left(u^{i}\right)$.

Step 3. If $\bar{\psi}_{\varepsilon_{j}}\left(u^{\prime}\right)>-\varepsilon_{j} / 2$, set $\varepsilon_{j+1}=\beta \varepsilon_{j}$, set $j=j+1$ and go to Step 2.

If $\bar{\psi}_{\varepsilon}\left(u^{i}\right) \leqslant-\varepsilon_{j} / 2$, proceed.

Step 4. Compute the smallest integer $k$ such that

$$
\begin{aligned}
& J^{0}\left(u^{i}+\beta^{k} \bar{w}_{e_{f}}^{u^{\prime}}\right)-J^{0}\left(u^{i}\right) \leqslant \alpha \beta^{k} \bar{\psi}_{\varepsilon_{1}}\left(u^{i}\right), \\
& J^{m}\left(u^{i}+\beta^{k} \bar{w}_{e_{1}}^{u^{\prime}}\right) \leqslant 0, \quad m=1, \ldots, p .
\end{aligned}
$$

Step 5. Set $u^{i+1}=u^{i}+\beta^{k} \bar{w}_{e_{j}}^{u^{i}}$. Set $i=i+1$ and go to Step 1 .

In what follows, our aim is to study the convergent property of the Algorithm 6.1. To be more precise, we shall show that the sequence of feasible controls generated by the feasible directions Algorithm 6.1 has an accumulation point in 
the weak* topology and that each of these accumulation points satisfies a necessary condition for optimality. Note that this weak* convergence development is similar to that given in [9] and [8]. However, there is no constraint on the state variables in [9], while the terminal inequality constraints on the state variables are only linear in [8]. In the present paper, we allow the terminal inequality constraints to be nonlinear. Furthermore, the technique used in this paper and that in [9] and [8] are completely different. To be more precise, a control parametrization technique is used in [9] and [8], while a feasible direction method is used here in the present paper.

Let

$$
\mathscr{D} \equiv\left\{u \in \mathscr{F}: \psi_{I_{0}}(u)=0\right\} .
$$

For any feasible control $u \notin \mathscr{D}$, let

$$
\bar{\varepsilon}(u) \equiv \max \left\{\varepsilon: \bar{\psi}_{\varepsilon}(u) \leqslant-\varepsilon / 2, \quad \varepsilon=\beta^{k} \varepsilon^{\prime}, \quad k=0,1, \ldots\right\} .
$$

and let $k(u)$ be the smallest non-negative integer satisfying (24) and (25).

LEMMA 6.1. For any subset $I$ of the index set $\{0,1, \ldots, l\}, \psi_{I}(\cdot)$ is upper semicontinuous with respect to the weak* topology. (In other words, if the sequence $\left\{u^{k}\right\}$ converges to $\bar{u}$ in the weak ${ }^{*}$ topology, then, for any given $\varepsilon>0$, there exists an integer $\hat{k}>0$ such that for all $\left.k>\hat{k}, \psi_{I}\left(u^{k}\right) \leqslant \psi_{I}(\bar{u})+\varepsilon\right)$.

Proof. The proof is similar to that given for Lemma 5.1 of [10], except that Remark 3.2 and $L_{\infty}$ topology of [10] are, respectively, replaced by Lemma 3.3 and the weak* topology in the proof of the present lemma.

Definition 6.1. Let $\varepsilon>0$ be given. Then, for any $m \in\{1, \ldots, l\}$, the corresponding terminal inequality constraint of (6) is said to be $\varepsilon$-active at $\bar{u} \in \mathscr{U}$ if $m \in I_{\varepsilon}(\bar{u})$. (For the definition of $I_{\varepsilon}(\bar{u})$, see (22).) (That is, if $J^{m}(\bar{u}) \equiv$ $g^{m}(x(\bar{u})(T)) \geqslant-\varepsilon$.)

LEMMA 6.2. Let $\left\{u^{i}\right\}$ be an infinite sequence in $\mathscr{F}$ converging to $\bar{u} \in \mathscr{D}$ in the weak* topology. Then, there exists an $\hat{\varepsilon}>0$ and an integer $i_{0}$ such that

$$
\bar{\varepsilon}\left(u^{i}\right) \geqslant \hat{\varepsilon}>0
$$

for all $i \geqslant i_{0}$. 
Proof. Following a similar approach as that given for Lemma 5.2 of [13], we get for each $u \in \mathscr{F} \backslash \mathscr{D}$,

$$
\bar{\varepsilon}(u) \geqslant \varepsilon(u),
$$

where

$$
\varepsilon(u) \equiv \max \left\{\varepsilon: \psi_{I_{\varepsilon}(u)}(u) \leqslant-\varepsilon, \varepsilon=\beta^{k} \varepsilon^{\prime}, k=0,1,2, \ldots\right\} .
$$

It remains to show that there exists an $\hat{\varepsilon}>0$ and an integer $i_{0}$ such that

$$
\varepsilon\left(u^{t}\right) \geqslant \hat{\varepsilon}>0
$$

for all $i \geqslant i_{0}$. For then by replacing $u$ by $u^{i}$ in (26), the conclusion of the lemma follows easily from (28) and (26).

To begin, we note that $\bar{u} \notin \mathscr{D}$. Thus, there exists a $\delta=\beta^{k_{1}} \varepsilon^{\prime}$ such that $\psi_{I_{0}(\bar{u})}(\bar{u})<-\delta$. Hence, there exists an $\varepsilon_{1}=\beta^{k_{2}} \varepsilon^{\prime}$ such that $\psi_{I_{\mathrm{e}}(\bar{u})}(\bar{u})<-\delta$ for all $\varepsilon \in\left[0, \varepsilon_{1}\right]$. Let $\varepsilon_{2}=\min \left[\varepsilon_{1}, \delta\right]$. Then, it is clear that

$$
\psi_{I_{\varepsilon_{2}}(\bar{u})}(\bar{u}) \leqslant \psi_{I_{\varepsilon_{1}}(\bar{u})}(\bar{u})<-\delta \leqslant-\varepsilon_{2},
$$

and hence

$$
\varepsilon(\bar{u}) \geqslant \varepsilon_{2}>0 .
$$

From Lemma 3.1 and (A3), it follows that there exists a positive integer $i_{1}$ such that for all $i \geqslant i_{1}, x\left(u^{\prime}\right)(T)$ is sufficiently close to $x(\bar{u})(T)$ for no additional constraints to become $\varepsilon(\bar{u})$-active. On this basis, we can deduce that, for all $i \geqslant i_{1}$,

$$
I_{e(\bar{u})}\left(u^{l}\right) \subset I_{e(\bar{u})}(\bar{u}) \equiv \bar{I}
$$

Thus, for all $i \geqslant i_{1}$,

$$
\psi_{I_{e(\bar{u})}\left(u^{\prime}\right)}\left(u^{i}\right) \leqslant \psi_{\bar{I}}\left(u^{i}\right) .
$$

Since $\psi_{I}(\cdot)$ is upper semicontinuous, there exists integer $i_{0} \geqslant i_{1}$ such that

$$
\psi_{l_{\ell(\bar{u})}\left(u^{l}\right)}\left(u^{i}\right) \leqslant \psi_{\bar{I}}\left(u^{i}\right) \leqslant \beta \psi_{\bar{I}}(\bar{u}) \leqslant-\beta \varepsilon(\bar{u})
$$

for all $i \geqslant i_{0}$. Therefore

$$
\psi_{I_{\beta e(u)}\left(u^{\prime}\right)}\left(u^{i}\right) \leqslant \psi_{I_{e(Q)}\left(u^{\prime}\right)}\left(u^{i}\right) \leqslant-\beta \varepsilon(\bar{u}),
$$

for all $i \geqslant i_{0}$. Thus, from (27), (30) and (29), we get

$$
\varepsilon\left(u^{\prime}\right) \geqslant \beta \varepsilon(\bar{u}) \geqslant \beta \varepsilon_{2} \equiv \hat{\varepsilon}>0,
$$

for all $i \geqslant i_{0}$. Hence (28) holds and the proof of the lemma is complete. 
LEMMA 6.3. Let $\left\{u^{i}\right\}$ be an infinite sequence of feasible controls converging to $\bar{u} \notin \mathscr{D}$ in the weak ${ }^{*}$ topology. Then, there exists $a \delta>0$ and positive integers $\hat{k}$ and $i_{0}$ such that for all $i \geqslant i_{0}$,

where

$$
\begin{aligned}
& k\left(u^{i}\right) \leqslant \hat{k}, \\
& J^{0}\left(\overline{u^{i}}\right)-J^{0}\left(u^{i}\right) \leqslant-\delta, \\
& J^{m}\left(\overline{u^{i}}\right) \leqslant 0, \quad m=1,2, \ldots, l,
\end{aligned}
$$

$$
\overline{u^{i}}=u^{i}+\beta^{k\left(u^{\prime}\right)} w_{\mathrm{e}\left(u^{\prime}\right)}^{u^{\prime}} .
$$

Proor. The proof is similar to that given for Proposition 3 of [7], except that Proposition 2 and 'the uniform continuity of $\nabla g^{0}(\cdot)$ on any $L_{\infty}^{m}[0,1]$ bounded set' in the proof of Proposition 3 of [7] are being replaced, respectively, by Lemma 6.2 and Lemma 3.2 (ii) in the proof of the present lemma.

COROllaRY 6.1. For any $u^{i} \in \mathscr{D} \backslash \mathscr{D}$, the computation of $\bar{\varepsilon}\left(u^{i}\right)$ and $k\left(u^{i}\right)$ in Algorithm 6.1 requires only a finite number of iterations.

THEOREM 6.1. If $u^{*} \in \mathscr{F}$ is an optimal control of the problem $P$, then $u^{*} \in \mathscr{D}$.

Proof. The proof is similar to that given for Corollary 5.2 of [13], except that Lemma 5.2 of [13] is replaced by Lemma 6.3 in the proof of the present theorem.

THEOREM 6.2. Let $\left\{u^{i}\right\}$ be a sequence of feasible controls generated by Algorithm 6.1. Then, either the sequence is finite, in which case the algorithm jams in the inner loops of Step 2 and Step 3, and the last element of the sequence is in $\mathscr{D}$, or the sequence is infinite and possesses at least one weak* accumulation point, where each of these weak* accumulation points is also in $\mathscr{D}$.

Proof. From Corollary 6.1, we note that the algorithm can jam only if the last $u^{i}$ is in $\mathscr{D}$. Therefore, it remains to prove the second part of the theorem. Suppose that $\left\{u^{i}\right\}$ is infinite. Since $\mathscr{F}$ is sequentially compact in the weak* topology (Remark 4.1), we conclude that the sequence possesses at least one weak* accumulation point in $\mathscr{F}$. Now, let $u^{*} \in \mathscr{F}$ be a $w^{*}$-accumulation point of $\left\{u^{i}\right\}$. Then, there exists a subsequence $\left\{u^{i(k)}\right\}$ of the sequence $\left\{u^{i}\right\}$ such that $u^{i(k)} \stackrel{w^{*}}{\rightarrow} u^{*}$ as $k \rightarrow \infty$. Since $\left\{J\left(u^{i}\right)\right\}$ is monotonically decreasing, it follows that

$$
J^{0}\left(u^{i(k+1)}\right)-J^{0}\left(u^{i(k)}\right) \leqslant J^{0}\left(u^{i(k)+1}\right)-J^{0}\left(u^{i(k)}\right) .
$$

Suppose that $u^{*}$ is not in $\mathscr{D}$. Then for sufficiently large $k$, we obtain from Lemma 6.3 and (31) that

$$
J^{0}\left(u^{i(k+1)}\right)-J^{0}\left(u^{i(k)}\right) \leqslant-\delta,
$$


where $\delta>0$ is a constant independent of integers $i(k), k=1,2, \ldots$ However, $J^{0}\left(u^{i(k)}\right)$ is a bounded and monotonically decreasing sequence of real numbers, and hence is convergent. Thus, by taking limit in (32), we obtain $0 \leqslant-\delta$. This is a contradiction and hence the proof is complete.

REMARK 6.1. From Remark 5.1 of [13], we recall that the convergence result of Theorem 6.2 is valid only if the initial control of Algorithm 6.1 is a feasible control.

REMARK 6.2. Since the problem considered in this paper is a special case of that considered in [10], an initial feasible control can be easily found by using the approach reported in Section 6 of [10]. More precisely, by using the control parametrization technique, this problem of obtaining an initial feasible control is reduced to the one amenable to the application of the algorithm in page 210 of [5].

\section{An illustrative example}

Consider the problem of minimizing

$$
J(u)=\frac{3}{2}[x(u)(3)]^{2}+\frac{1}{2} \int_{0}^{3}[u(t)]^{2} d t
$$

subject to the delay-differential equation

$$
\dot{x}(t)=x(t-1)+u(t), \quad t \in(0,3],
$$

with the initial condition

$$
x(t)=1, \quad t \in[-1,0],
$$

and the terminal inequality constraints

$$
\begin{aligned}
& g^{1}(x(u)(3)) \equiv[x(u)(3)]^{2}-0.03 \leqslant 0 \\
& g^{2}(x(u)(3))=0.06+[x(u)(3)]^{2} \leqslant 0
\end{aligned}
$$

where $u$ is the control function with values in $[-3,3]$.

Let the above problem be denoted by $Q$. This problem is adapted from that considered in Example 4.1 of [2], page 189. More precisely, there are no control constraints and terminal inequality constraints in the original problem. Let the original problem be denoted by $\bar{Q}$. From [2], we know that the optimal control of the unconstrained problem $\bar{Q}$ is

$$
u^{*}(t)= \begin{cases}\delta\left(-(t-2)^{2} / 2-3 / 2\right), & t \in[0,1] \\ \delta(t-3), & t \in[1,2] \\ -\delta, & t \in[2,3]\end{cases}
$$


where $\delta$ is given (approximately) by

$$
\delta=0.56231 .
$$

The optimal cost is approximately 1.7338 .

Note that $u^{*}$ satisfies the control constraints and that both of the terminal inequality constraints are also satisfied when $u=u^{*}$. Thus, $u^{*}$ is also the optimal control of the problem $Q$.

The problem $Q$ is to be solved in two stages as described below. For both stages, the combination of the fourth order Runge-Kutta integration scheme together with the method of steps is used to integrate the system forward in time and the adjoint system backward in time, both over the interval $[0,3]$, which is divided into 300 netpoints.

\section{STAGE 1: To find a feasible control.}

Let the interval $[0,3]$ be divided into 5 subintervals such that the length of each interval is equal to 0.6. Using the initial control equal to zero throughout each of the subintervals (note that this control is not a feasible control because $g^{2}(x(0)(3))=37.9678>0$ ), a feasible control $u^{0}$ was found by using the procedure mentioned in Remark 6.2. It takes 70 iterations of the algorithm of [5], where the parameters $a$ and $b$ are chosen to be 0.01 and 0.5 , respectively. This feasible control $u^{0}$ is

$$
u^{0}(t)= \begin{cases}-1.6886, & t \in[0,0.6) \\ -1.1728, & t \in[0.6,1.2) \\ -0.8307, & t \in[1.2,1.8) \\ -0.5723, & t \in[1.8,2.4) \\ -0.5538, & t \in[2.4,3.0)\end{cases}
$$

\section{STAGE 2: To find an optimal control.}

Using $u^{0}$ as the initial feasible control and the parameters $(\alpha, \beta, \varepsilon)=$ $(0.2,0.8,0.01)$ (respectively $(\alpha, \beta, \varepsilon)=(0.2,0.8,0.005))$, the 'optimal' cost obtained by the combination of Algorithm 5.1 and Algorithm 6.1 after 70 (respectively 100) iterations is 1.7339 (respectively 1.7341). These values are extremely close to the true optimal cost 1.7338 .

For the case $\varepsilon=0.01$, the constraint (33b) was $\varepsilon$-active on 57 occasions throughout the 70 iterations. For the case $\varepsilon=0.005$, the constraint (33b) was $\varepsilon$-active on 27 occasions throughout the 100 iterations. Numerical results are listed in Tables 7.1 and 7.2. Graphs of $u^{70}(t)$ with $(\alpha, \beta, \varepsilon)=(0.2,0.8,0.01)$ and the true optimal control are plotted in Figure 1 and Figure 2, respectively. 


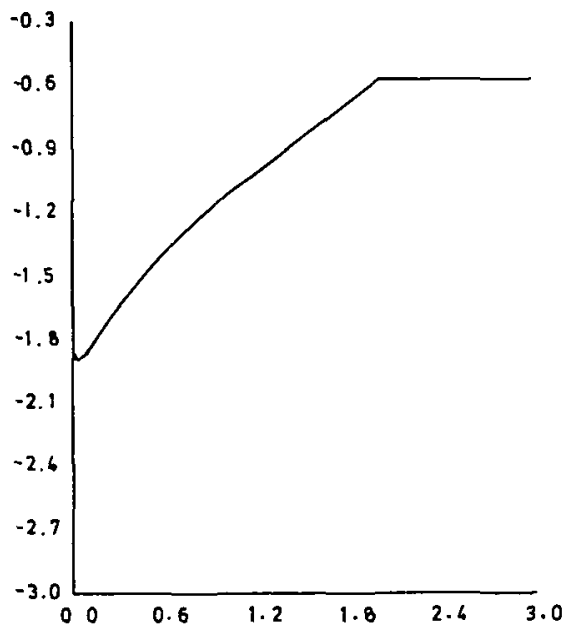

Figure 1. $u^{70}(t) \quad \alpha=0.2, \beta=0.8, \epsilon=0.01$

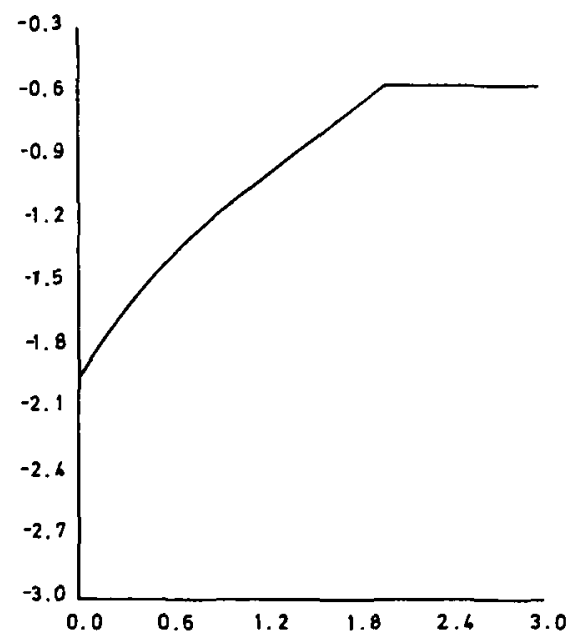

Figure 2. The true optimal control. 
TABLE 7.1 Numerical Results

$\left(\begin{array}{c}\alpha=0.2, \quad \beta=0.8, \quad \varepsilon=0.01 \\ \text { Initial Control is defined by (34) }\end{array}\right)$

\begin{tabular}{|c|c|}
\hline$i$ & $J\left(u^{\prime}\right)$ \\
\hline 10 & 1.7404 \\
20 & 1.7376 \\
30 & 1.7350 \\
40 & 1.7342 \\
50 & 1.7341 \\
60 & 1.7339 \\
70 & 1.7339 \\
\hline
\end{tabular}

TABLE 7.2 Numerical Results

$$
\left(\begin{array}{cc}
\alpha=0.2, \quad \beta=0.08 \quad \varepsilon=0.005 \\
\text { Initial Control is defined by (34) }
\end{array}\right)
$$

\begin{tabular}{|r|r|}
\hline$i$ & $J\left(u^{\prime}\right)$ \\
\hline 10 & 1.7406 \\
20 & 1.7380 \\
30 & 1.7354 \\
40 & 1.7348 \\
50 & 1.7374 \\
60 & 1.7346 \\
70 & 1.7345 \\
80 & 1.7342 \\
90 & 1.7341 \\
100 & 1.7341 \\
\hline
\end{tabular}

\section{Acknowledgement}

The authors wish to thank Dr. B. D. Craven in the Department of Mathematics at the University of Melbourne, for his most valuable comments and suggestions.

\section{References}

[1] N. U. Ahmed and K. L. Teo, Optimal control of distributed parameter systems (North-Holland, New York, 1981).

[2] H. T. Banks and J. A. Burns, "Hereditary control problems: numerical methods based on averaging approximations", SIAM J. Control Optim. 16 (1978), 169-208. 
[3] B. D. Craven, Mathematical programming and control theory (Chapman and Hall, London, 1978).

[4] N. D. Georganas, "Optimal control for a class of hereditary systems”, Ph.D. Thesis, University of Ottawa, Ottawa, Canada, 1970.

[5] D. Q. Mayne, E. Polak and A. J. Heunis, "Solving nonlinear inequalities in a finite number of iterations", J. Optim. Theory Appl. 33 (1981), 207-221.

[6] E. Polak, Computational methods in optimization (Academic Press, New York, 1971).

[7] E. Polak and D. Q. Mayne, "A feasible directions algorithm for optimal control problems with control and terminal inequality constraints", IEEE Trans. Automat. Control AC-22 (5) (1977), $741-751$.

[8] K. L. Teo and R. S. Womersley, "A control parametrization algorithm for optimal control problems involving linear systems and linear terminal inequality constraints", Numer. Funct. Anal. Optim. 6 (1983), 291-313.

[9] K. L. Teo, K. H. Wong and D. J. Clements, "Optimal control computation for linear time-lag systems with linear terminal constraints", J. Optim. Theory Appl. 44 (1984), 509-526.

[10] K. L. Teo, K. H. Wong and D. J. Clements, "A feasible directions algorithm for time-lag optimal control problems with control and terminal inequality constraints", J. Optim. Theory Appl. 46 (1985), 295-317.

[11] K. L. Teo and Z. S. Wu, Computational methods for optimizing distributed systems (Academic Press, Orlando, 1984).

[12] K. H. Wong and K. L. Teo, "A conditional gradient method for a class of time-lag optimal control problems", J. Austral. Math. Soc. Ser. B 25 (1984), 518-537.

[13] Z. S. Wu and K. L. Teo, "A computational algorithm for a distributed optimal control problem of parabolic type with terminal inequality constraints", J. Optim. Theory Appl. 43 (1984), 457-476. 